\title{
Neutrino quasielastic interaction and nuclear dynamics
}

\author{
M. Martini \\ CEA/DAM/DIF, F-91297 Arpajon, France \\ M. Ericson \\ Université de Lyon, Univ. Lyon 1, CNRS/IN2P3, IPN Lyon, F-69622 Villeurbanne Cedex, France and \\ Physics Department, Theory Unit, CERN, CH-1211 Geneva, Switzerland \\ G. Chanfray \\ Université de Lyon, Univ. Lyon 1, CNRS/IN2P3, IPN Lyon, F-69622 Villeurbanne Cedex, France \\ (Received 10 October 2011; revised manuscript received 29 October 2011; published 28 November 2011)
}

\begin{abstract}
We investigate the double differential neutrino-carbon quasielastic cross sections as measured by the MiniBooNE experiment. Our present treatment incorporates relativistic corrections in the nuclear response functions and includes the multinucleon component. We confirm our previous conclusion that it is possible to account for all the data without any modification of the axial mass. We also introduce the $Q^{2}$ distribution for charged and neutral current. The data point at a sizable multinucleon component beside the genuine quasielastic peak. They are also indicative of the collective character of the nuclear response, of interest for hadronic physics.
\end{abstract}

DOI: 10.1103/PhysRevC.84.055502

PACS number(s): 25.30.Pt, 13.15.+g, 24.10.Cn

\section{INTRODUCTION}

Recent data on neutrino-nucleus scattering have improved our understanding of the neutrino-nucleus interaction, needed for neutrino oscillation experiments where nuclear targets are involved. Among the results on partial cross sections, the quasielastic one turns out to be very important [1,2]. An outcome of these data was the display of an "anomaly" in the quasielastic cross section on ${ }^{12} \mathrm{C}$. This quantity can be fitted by a relativistic Fermi gas model only at the price of a modification of the axial form factor with an axial mass $M_{A}=1.35 \mathrm{GeV}$, instead of the usual value $M_{A}=1.03 \mathrm{GeV}$ as measured in deuteron bubble chamber experiments. For nuclear physicists accustomed to the complexity of the manybody nuclear system, this anomaly is likely to reflect the many-body aspect of the problem. Indeed we have pointed out $[3,4]$ that, depending on the detection method, certain types of inelastic events can simulate quasielastic ones. This is the case for interactions leading to a final state with two or more nucleons ejected, if a quasielastic event is defined as one with only a muon in the final states, as in MiniBooNE. Multinucleon processes occur by nuclear correlations, with or without Delta excitation. We have argued that this is the likely explanation of the anomaly showing that an evaluation can account for the excess cross section without any modification of the axial mass. After this suggestion, a number of articles [5-11] have discussed the problem of multinucleon emission and whether it could account for the anomaly, with various conclusions, critical or supportive of our result.

Our previous works only dealt with the quasielastic cross section as a function of the neutrino energy. In the comparison with the experimental data, the uncertainties linked to the fact that the neutrino spectrum is broad are of experimental origin because the extraction of the energy dependence of the cross section involves a reconstruction of the neutrino energy whereas in the theoretical evaluation the neutrino energy is just an input. In the present work we discuss the double differential cross section. This is a directly measured quantity, free from the uncertainty of neutrino energy reconstruction. However, there remains an uncertainty on the theoretical side because the measured double differential cross section refers to the broad spectrum of neutrino energies. The theoretical predictions imply a convolution on this spectrum, which could be a source of error. Nevertheless, a good agreement with theory for the double differential cross section speaks in favor of the importance of the role of multinucleon emission process. In the present article we will also discuss the role played by relativistic kinematics. The momenta and energies involved in these neutrino reactions are rather large. For the MiniBooNE experiment the neutrino energy extends to $\simeq 2 \mathrm{GeV}$ and the ejected nucleon kinetic energy in a quasielastic process can be a few hundred $\mathrm{MeV}$ making a nonrelativistic approximation questionable. Indeed, it was pointed out that conclusions on the role of the multinucleon process are doubtful within a nonrelativistic framework [7]. It is one of the aims of this work to answer these criticisms. To improve our description we introduce in the present work relativistic modifications of the nuclear response, as proposed in $[12,13]$. To single out their influence we keep for all the remainder of the description the same input parameters that we used in our previous work, in particular, as concerns the description of the two particle-two hole $(2 p-2 h)$ processes, for which we use our parametrization deduced from the work of Alberico et al. [14] on the $2 p-2 h$ contribution to the transverse response. Remember that we also have a $3 p-3 h$ contribution, taken from Ref. [15]. All together we denote the sum by $n p-n h$. We also keep the same values of the parameters of the particle-hole $(p-h)$ force which governs the collective aspect of the nuclear response via the random phase approximation (RPA). We will show that although the relativistic treatment improves the description of the double differential cross section it is nearly without influence on the 


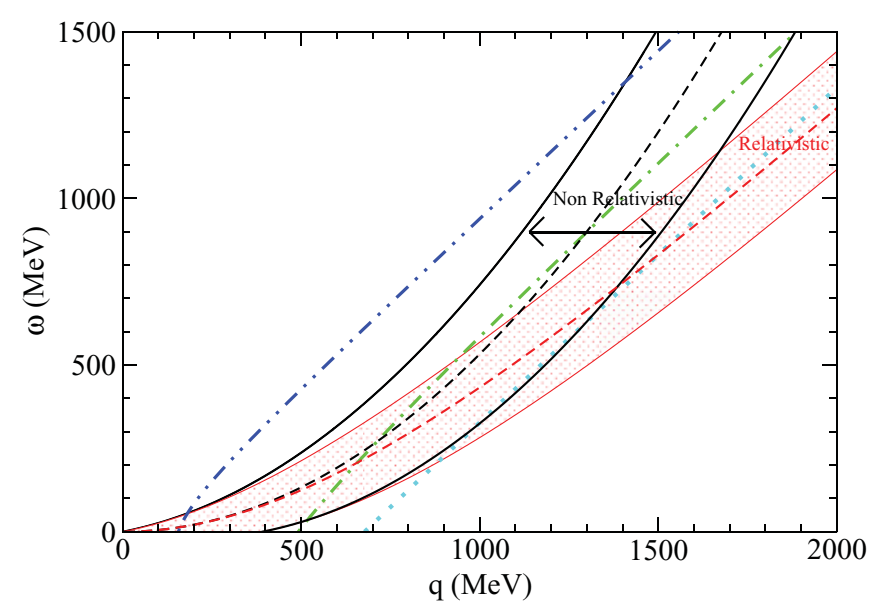

FIG. 1. (Color online) Regions of the quasielastic response of a Fermi gas. For relativistic kinematics, see shaded area (red) delimited by the two corresponding continuous lines. In the nonrelativistic case the horizontal arrow shows the two limiting lines (black). The central dashed lines show the position of the quasielastic peak in the two cases. The remnant three lines represent the neutrino hyperbolas defined by Eq. (2) for a muon kinetic energy $T_{\mu}=250 \mathrm{MeV}$ and three muon emission angles: $\cos \theta=0.9$ [dot-dot-dashed line (blue)], $\cos \theta=0$ [dot-dashed line (green)], and $\cos \theta=-0.9$ [dotted line (turquoise)].

integrated quasielastic cross sections. Our previous conclusion on the role played by the multinucleon processes in the axial anomaly is not an artifact of the nonrelativistic treatment of our earlier works. Then we give the single differential cross sections, that is, integrated over the muon energy, or the muon angle, and the $Q^{2}$ distribution not only for charged current (CC) but also for neutral current (NC).

\section{ANALYSIS OF DIFFERENTIAL CROSS SECTIONS}

For a given "quasielastic" event the muon energy $E_{\mu}$ (or kinetic energy $T_{\mu}$ ) and its emission angle $\theta$ are measured. The neutrino energy $E_{v}$ is unknown. In the experimental analysis a specific assumption is made concerning the quasielastic character of the one muon events. Nuclear cross sections are naturally expressed in terms of the nuclear responses, functions of the energy and momentum transferred to the nuclear system, $\omega=E_{v}-E_{\mu}$, and $q=|\vec{q}|=\left|\vec{p}_{v}-\vec{p}_{\mu}\right|$. These are the natural variables but they are not the measured quantities. For each value of $E_{\mu}$ and $\theta$ several values of $\omega$, hence of $E_{\nu}=E_{\mu}+\omega$, are possible. The expression of the double differential cross section in terms of the measured quantity is

$$
\begin{aligned}
& \frac{d^{2} \sigma}{d T_{\mu} d \cos \theta} \\
& \quad=\frac{1}{\int \Phi\left(E_{\nu}\right) d E_{\nu}} \int d E_{v}\left[\frac{d^{2} \sigma}{d \omega d \cos \theta}\right]_{\omega=E_{\nu}-E_{\mu}} \Phi\left(E_{\nu}\right) .
\end{aligned}
$$

In the numerical evaluations we use the neutrino flux $\Phi\left(E_{v}\right)$ from Ref. [1].

The cross section of the right-hand side of Eq. (1), as expressed in terms of the nuclear responses [3], is nonvanishing

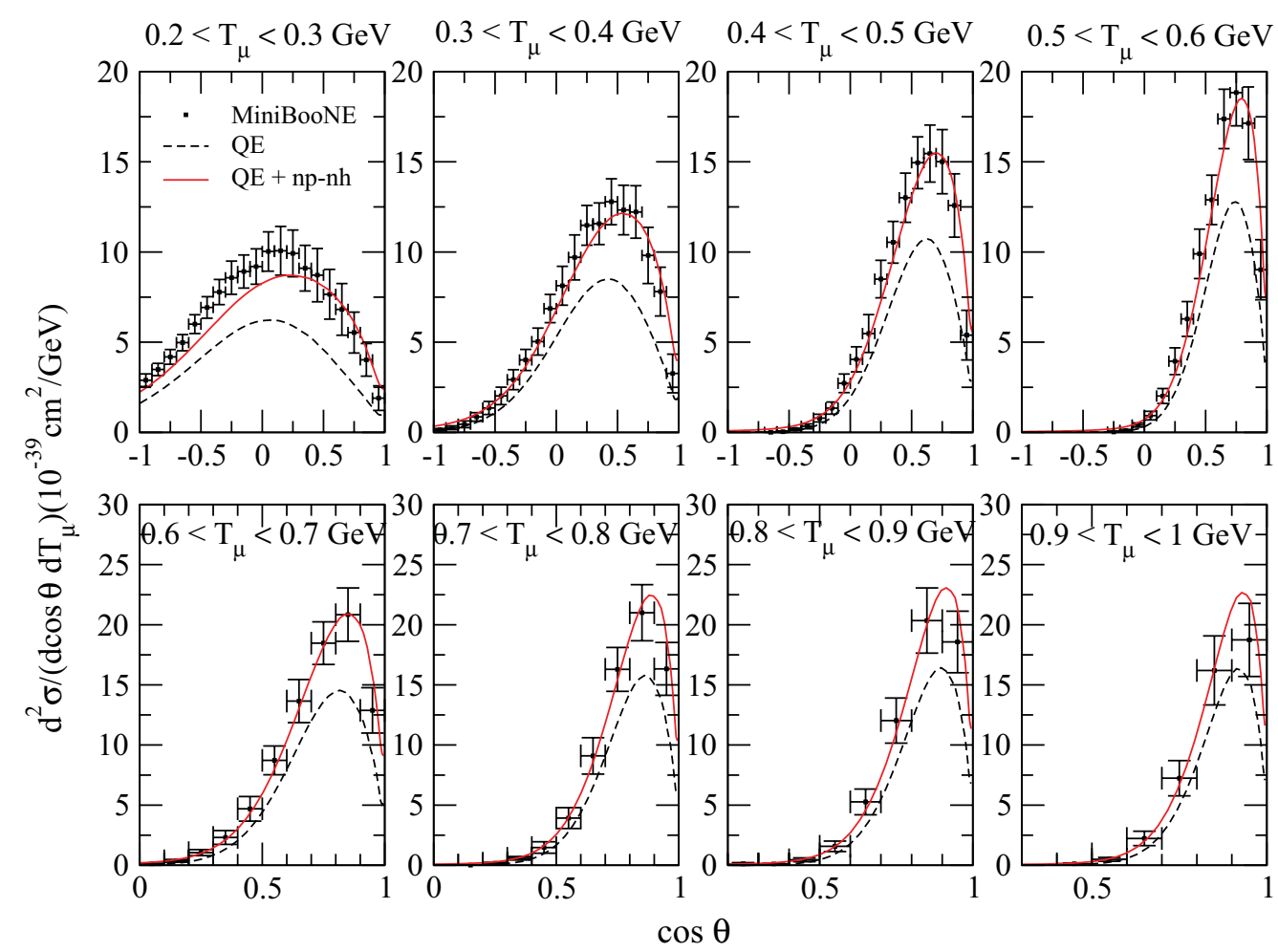

FIG. 2. (Color online) MiniBooNE flux-averaged CC "quasielastic" $v_{\mu^{-}}{ }^{12} \mathrm{C}$ double differential cross section per neutron for several values of muon kinetic energy as a function of the scattering angle. (Dashed curve) Pure quasielastic ( $1 p-1 h)$ cross section calculated in RPA, (solid curve) with the inclusion of $n p-n h$ component. The experimental MiniBooNE points are taken from Ref. [1]. 


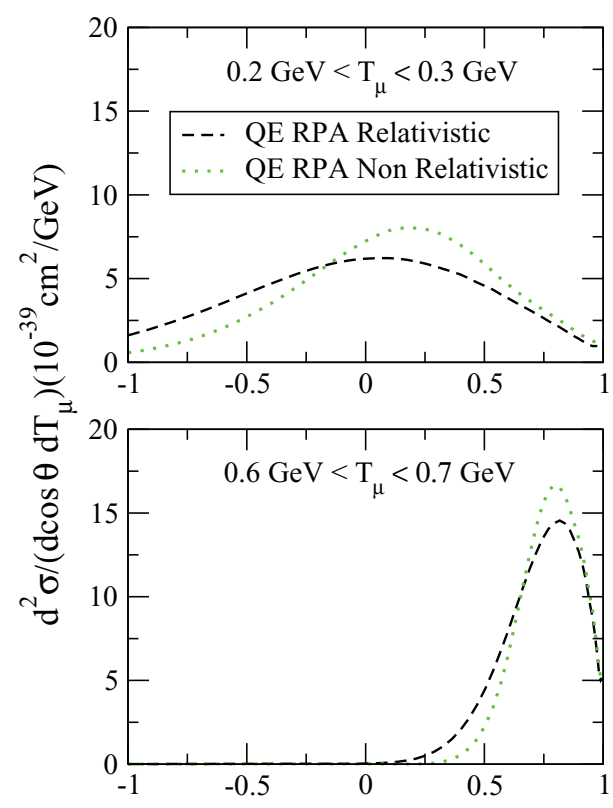

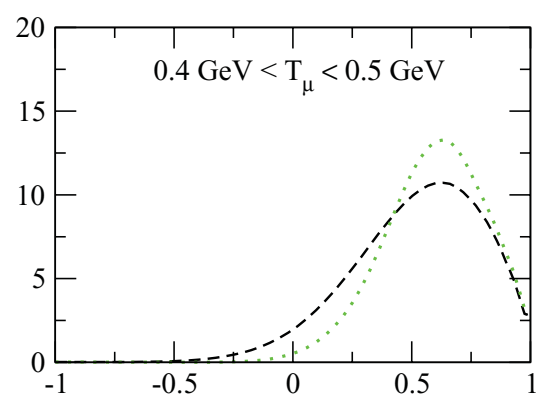

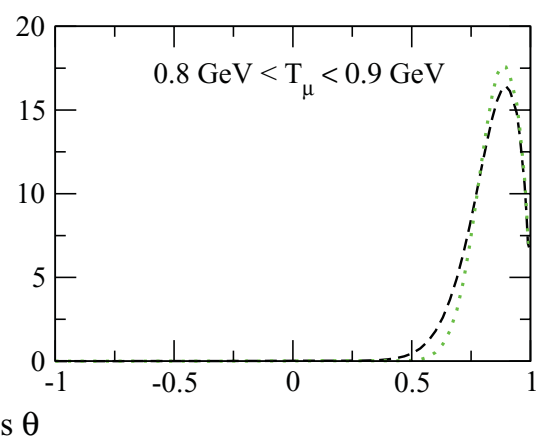

FIG. 3. (Color online) MiniBooNE flux-averaged $\mathrm{CC}$ genuine quasielastic $v_{\mu^{-}}{ }^{12} \mathrm{C}$ double differential cross section per neutron for several values of muon kinetic energy as a function of the scattering angle and calculated in RPA. (Dashed curve) With relativistic corrections; (dotted curve) without relativistic corrections. in the regions of the $\omega$ and $q$ plane where the responses are nonzero, regions defined in the following. For a very dilute Fermi gas the region of response is restricted to the line of the quasielastic peak, namely $\omega=q^{2} /\left(2 M_{N}\right)$ in the nonrelativistic case and $\omega=Q^{2} /\left(2 M_{N}\right)$ for the relativistic kinematics. At finite density there is a spreading caused by the Fermi motion and the region of response is delimited, when $q>2 k_{F}$, by the two lines $\omega_{ \pm}=\left(q^{2} \pm 2 q k_{F}\right) /\left(2 M_{N}\right)$, for the nonrelativistic case and by $\omega_{ \pm}=\sqrt{q^{2} \pm 2 q k_{F}+M_{N}^{2}}-M_{N}$, for the relativistic one. For $q<2 k_{F}$, the lower bound is the $\omega=0$ axis in both cases. The two lines delimiting the regions of response are represented in Fig. 1, together with the central one which shows the position of the quasielastic peak, where the response has its maximum, both in the relativistic and nonrelativistic cases.

To illustrate how these regions are explored in neutrino reactions we write the squared four-momentum transfer in terms of the lepton observables,

$$
\begin{aligned}
Q^{2}= & q^{2}-\omega^{2}=4\left(E_{\mu}+\omega\right) E_{\mu} \sin ^{2} \frac{\theta}{2}-m_{\mu}^{2} \\
& +2\left(E_{\mu}+\omega\right)\left(E_{\mu}-p_{\mu}\right) \cos \theta
\end{aligned}
$$

with $p_{\mu}=\left|\vec{p}_{\mu}\right|$. For a given set of observables $E_{\mu}$ and $\theta$ this relation defines a hyperbola in the $\omega$ and $q$ plane [16]. The asymptotes are parallel to the $\omega=q$ line and the intercept of the curves with the $\omega=0$ axis occurs at a value of the momentum,

$$
q_{\mathrm{int}}^{2}=4 E_{\mu}^{2} \sin ^{2} \frac{\theta}{2}-m_{\mu}^{2}+2 E_{\mu}\left(E_{\mu}-p_{\mu}\right) \cos \theta \simeq 4 E_{\mu}^{2} \sin ^{2} \frac{\theta}{2},
$$

where the second expression is obtained by neglecting the muon mass. With increasing $E_{\mu}$ or increasing angle, this point shifts away from the origin. The neutrino cross section for a given $T_{\mu}$ and $\theta$ explores the nuclear responses along the corresponding hyperbola. In Fig. 1 the quasielastic peak lines are shown together with some examples of hyperbolas. This figure illustrates the problems associated with the nonrelativistic kinematics: The intercept of the hyperbolas with the quasielastic line disappears at large angles, which does not occur in the relativistic case. There can also be two intercepts, which is not realistic either. To suppress the pathologies of the nonrelativistic dynamics and to implement the relativistic corrections we use results from quasielastic electron scattering studies [12,13]. They showed that a good approximation to simulate a relativistic treatment starting from a nonrelativistic frame is obtained with the substitution $\omega \rightarrow \omega\left(1+\frac{\omega}{2 M_{N}}\right)$ in the nuclear responses (which insures the right position of

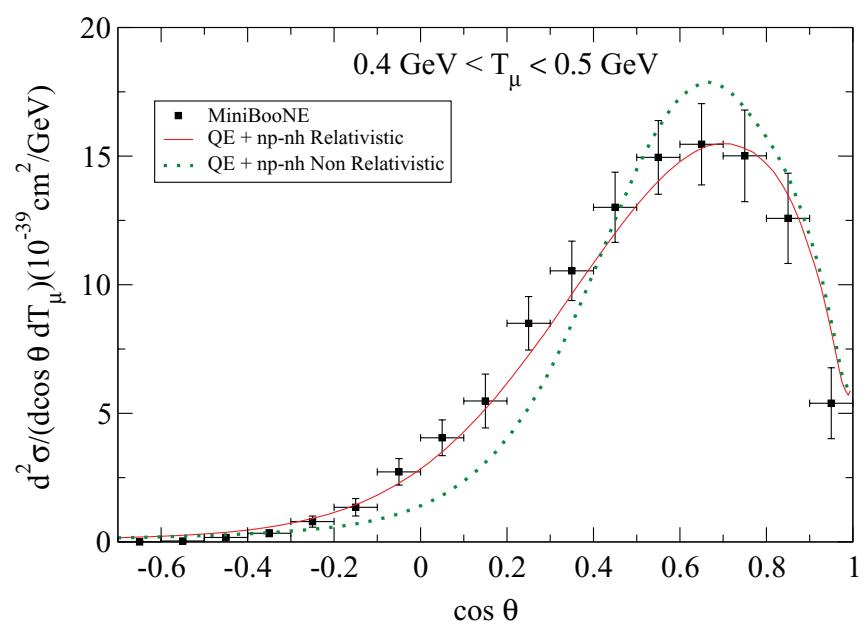

FIG. 4. (Color online) MiniBooNE flux-averaged CC "quasielastic" $v_{\mu^{-}}{ }^{12} \mathrm{C}$ double differential cross section per neutron for $0.4 \mathrm{GeV}$ $<T_{\mu}<0.5 \mathrm{GeV}$ as a function of the scattering angle calculated in RPA with the inclusion of the $n p-n h$ component. (Solid curve) With relativistic corrections; (dotted curve) without relativistic corrections. 


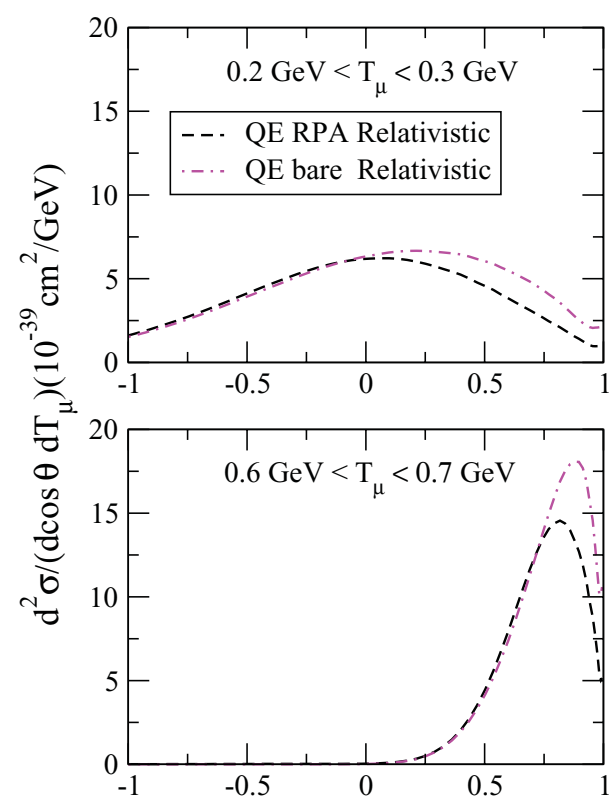

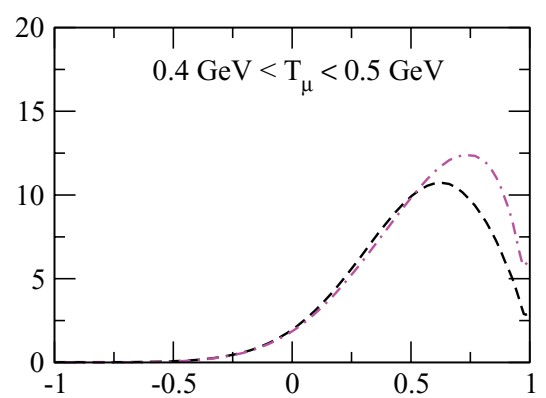

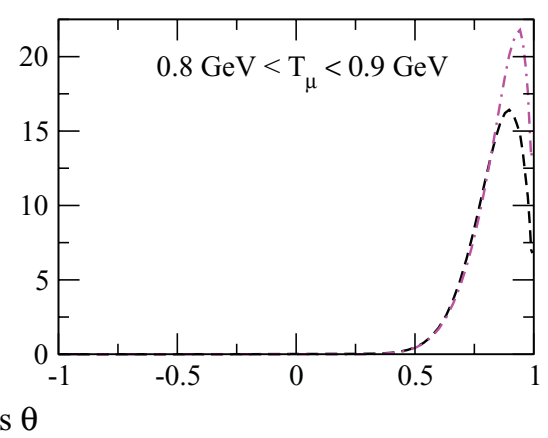

FIG. 5. (Color online) MiniBooNE flux-averaged $\mathrm{CC}$ genuine quasielastic $v_{\mu}{ }^{-12} \mathrm{C}$ double differential cross section per neutron for several values of muon kinetic energy as a function of the scattering angle. (Dashed curve) Calculated in RPA; (dot-dashed curve) bare. the quasielastic peak), and by multiplying the responses by $\left(1+\frac{\omega}{M_{N}}\right)$. Our present evaluations use these recipes and unless specified otherwise the curves of this article are calculated in this framework. Now in a realistic approach of the nuclear dynamics with correlations the nuclear region of response is not restricted to the Fermi motion band around the quasielastic line (as in Fig. 1) but it covers the whole $\omega$ and $q$ plane from multinucleon emission. As a consequence, for a given set of values of $E_{\mu}$ and $\theta$, all values of the energy transfer $\omega$, hence of the neutrino energy, $E_{v}=E_{\mu}+\omega$, contribute and one explores the full energy spectrum of neutrinos above the muon energy.

The results of our present evaluation with the relativistic corrections of the double differential cross section are displayed in Fig. 2, with and without the inclusion of the $n p-n h$ component and compared to the experimental data. This evaluation, like all those in this article, is done with the free value of the axial mass. The agreement is quite good in all the measured ranges once the multinucleon component is incorporated. Similar conclusions have been recently reported in Ref. [9]. The relativistic corrections are significant, as illustrated in Fig. 3 which compares the two approaches for the genuine quasielastic contributions. The relativistic treatment, which suppresses the kinematical pathologies, improves the description, in particular, in the backward direction. This is illustrated in Fig. 4 in the case $0.4 \mathrm{GeV}<T_{\mu}<0.5 \mathrm{GeV}$ in which the $2 p-2 h$ component was added for comparison with data. The good agreement with data of Fig. 2 is absent in the nonrelativistic case.

Our responses are described, as in our previous works $[3,4]$, in the framework of random phase approximation. Its role is shown in Figs. 5 and 6 where the double differential cross sections as a function of $\cos \theta$ or $T_{\mu}$ are displayed with and without RPA. The RPA produces a quenching and some shift toward larger angles or larger $T_{\mu}$. In Fig. 6 we present the comparison with data adding the $n p-n h$ to the genuine QE with or without RPA. The fit is significantly better in the RPA framework, reflecting the collective character of the nuclear response. The RPA quenching of the cross sections results from the repulsive nature of the $p$ - $h$ force, embodied in the Landau-Migdal parameter $g^{\prime}$. A large part of this quenching arises from the mixing of the $p$ - $h$ states with $\Delta$-hole ones. This is the Lorentz-Lorenz effect, which concerns exclusively the spin isospin response, hence the axial or magnetic matrix elements. In the graphical illustration of the response, the Lorentz-Lorenz effect on the quasielastic one is illustrated in Fig. 7. Figure 6 shows the dominance of

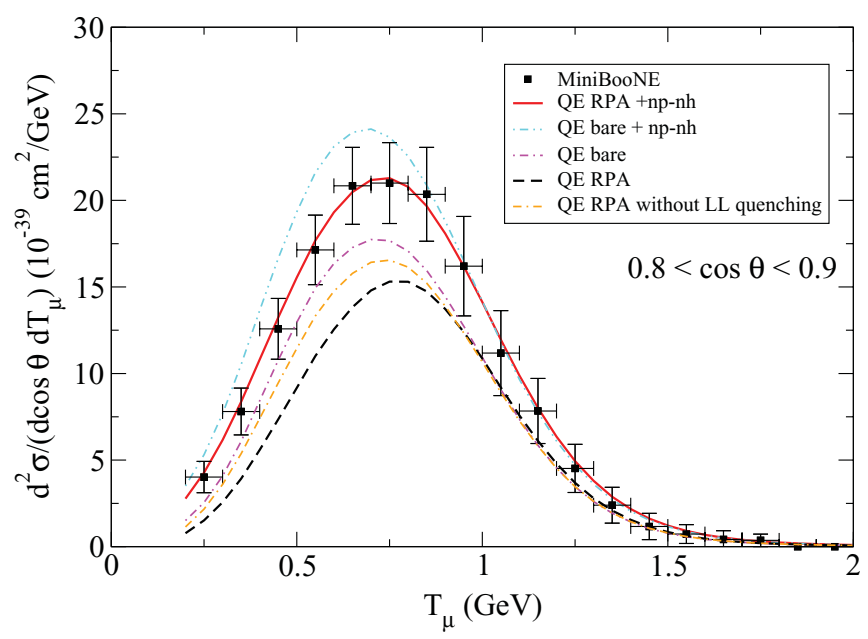

FIG. 6. (Color online) MiniBooNE flux-averaged CC quasielastic $v_{\mu^{-}}{ }^{12} \mathrm{C}$ double differential cross section per neutron for $0.8<\cos \theta<$ 0.9 as a function of the muon kinetic energy. (Dashed curve) Pure quasielastic calculated in RPA; (solid curve) RPA quasielastic with the inclusion of $n p-n h$ component; (dot-dot-dashed) bare quasielastic with the inclusion of $n p-n h$ component; (dot-dashed curve) bare quasielastic; (dot-dashed-dashed) RPA quasielastic without the Lorentz-Lorenz (LL) quenching. 


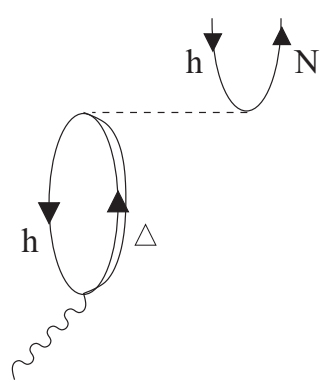

FIG. 7. Lowest-order contribution of Lorentz-Lorenz effect on the quasielastic response. The wiggled lines represent the external probe, and the dashed lines an effective interaction between nucleon hole and $\Delta$ hole.

the Lorentz-Lorenz quenching effect in the RPA quenching of the neutrino cross section. The Lorentz-Lorenz effect was first predicted for the axial $\beta$-decay matrix elements [17]; in this case its existence was controversial. A similar concept was shown by Alberico et al. [18] to apply to the spin-isospin nuclear responses in the region of the quasielastic peak at finite momenta. With the introduction in Ref. [18] of the collective character of the nuclear responses via an RPA treatment, the Lorentz-Lorenz mixing effect naturally appeared. However, on the experimental side, the transverse (magnetic) part of the inclusive electron scattering data have not clearly established the collective nature of this response nor the Lorentz-Lorenz quenching, because they are mixed with other effects. It is interesting that they seem to show up in neutrino reactions. It is, in fact, the integration over the energy transferred to the nuclear system which is contained in these cross sections which allows more easily the emergence of their gross features, an unexpected outcome of these data.

In the following we analyze the single differential cross sections, integrated over one of the two independent variables,

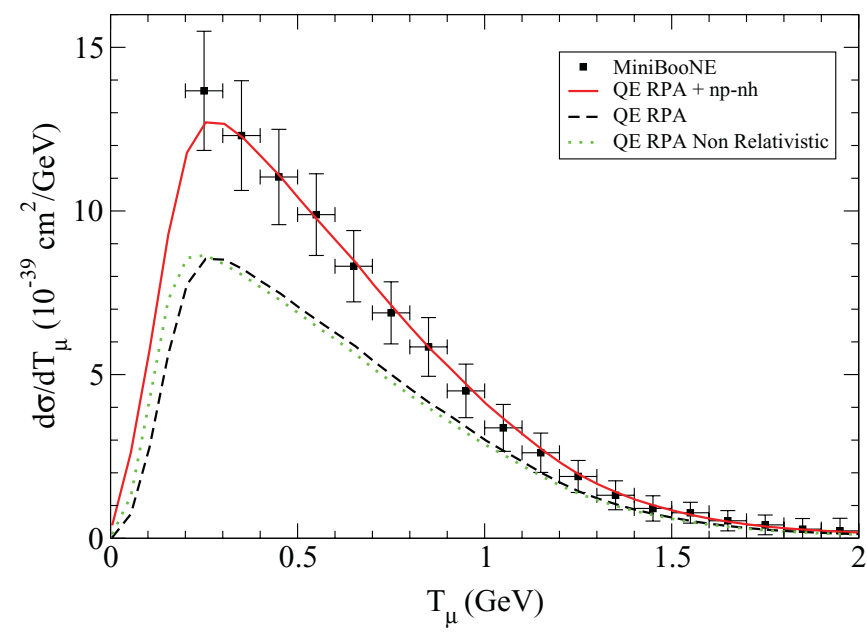

FIG. 8. (Color online) MiniBooNE flux-averaged CC "quasielastic" $v_{\mu^{-}}{ }^{12} \mathrm{C}$ differential cross section per neutron as a function of the muon kinetic energy. (Dashed curve) Pure quasielastic $(1 p-1 h)$ cross section; (solid curve) with the inclusion of $n p-n h$ component; (dotted line) pure quasielastic with nonrelativistic kinematics. The experimental MiniBooNE points are taken from Ref. [1].

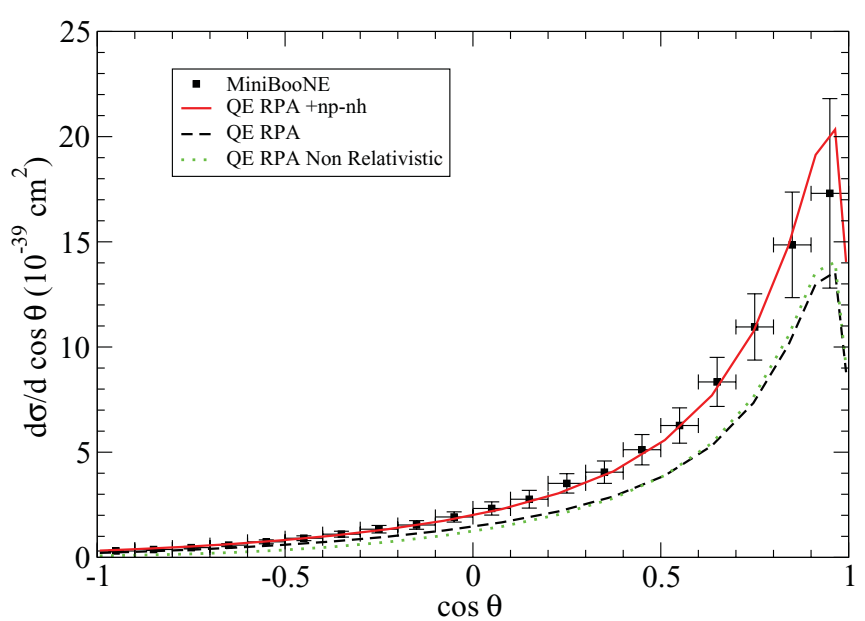

FIG. 9. (Color online) MiniBooNE flux-averaged CC "quasielastic" $v_{\mu}{ }^{-12} \mathrm{C}$ differential cross section per neutron as a function of the muon scattering angle. Note that to compare with data the integration is performed over the muon kinetic energies $0.2 \mathrm{GeV}<T_{\mu}<2.0 \mathrm{GeV}$. (Dashed curve) Pure quasielastic (1p-1h) cross section; (solid curve) with the inclusion of $n p-n h$ component; (dotted line) pure quasielastic with nonrelativistic kinematics. The experimental MiniBooNE points are taken from Ref. [1].

muon energy or angle, with and without the $n p-n h$ contribution. These are displayed in Figs. 8 and 9. The agreement of the integrated cross sections with the data is good in both cases if the multinucleon contribution is included, as displayed in Figs. 8 and 9. Here, contrary to the previous case, the relativistic corrections have a small influence. A single integration nearly washes out the relativistic effects. This is $a$ fortiori true for the total cross section.

Finally we show in Fig. 10 the single differential cross section with respect to $Q^{2}$, which was historically of interest for the determination of the axial form factor. Figure 10 shows

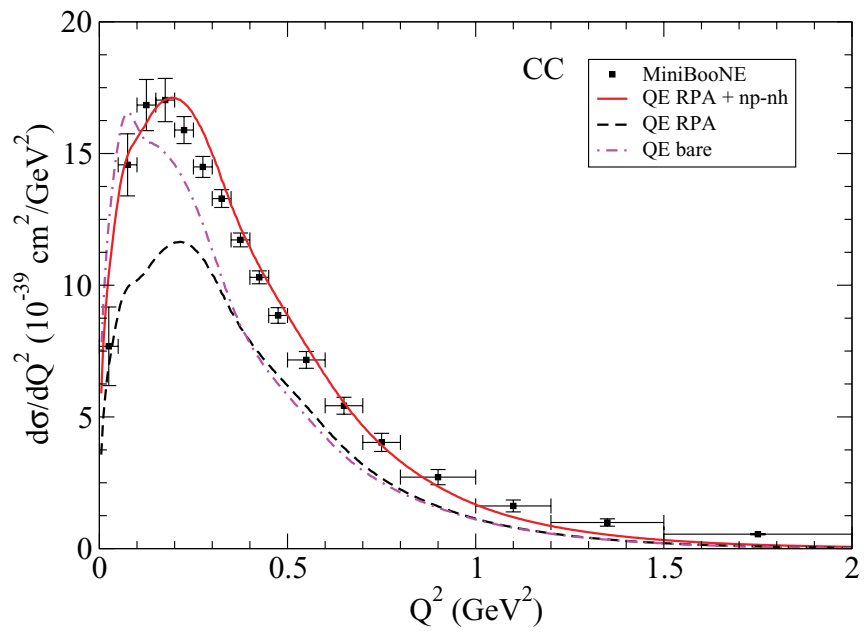

FIG. 10. (Color online) MiniBooNE flux-averaged CC $Q^{2}$ distribution per neutron. (Dashed curve) Pure quasielastic $(1 p-1 h)$; (solid curve) with the inclusion of $n p-n h$ component; (dot-dashed line) bare distribution. The experimental MiniBooNE points are taken from Ref. [1]. 


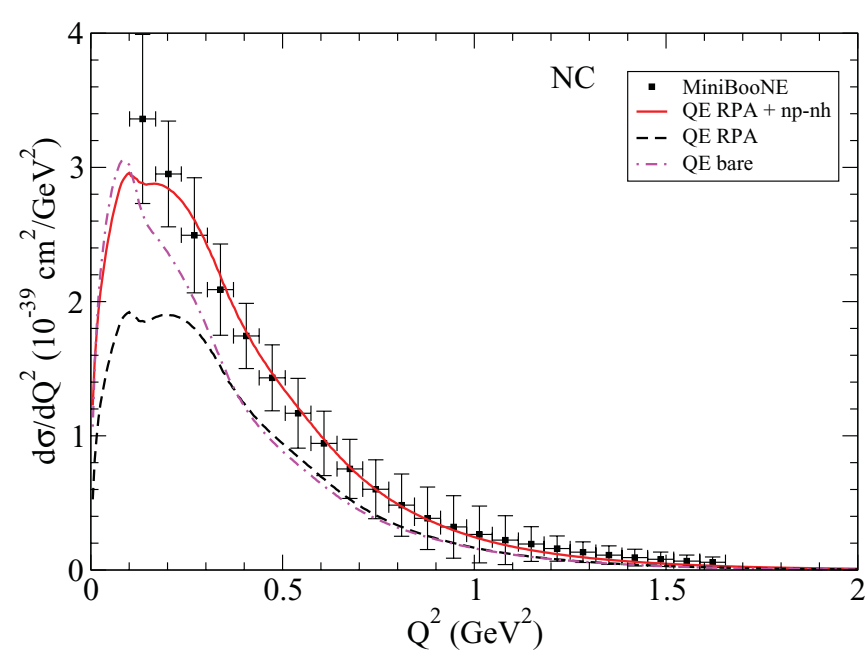

FIG. 11. (Color online) MiniBooNE flux-averaged NC $Q^{2}$ distribution per nucleon. (Dashed curve) Pure quasielastic (1p-1h); (solid curve) with the inclusion of $n p-n h$ component; (dot-dashed line) bare distribution. The experimental MiniBooNE points are taken from Ref. [2].

that here also the fit is good without any modifications of the axial mass but provided the multinucleon component is incorporated. In this figure the existence of a large $Q^{2}$ region, $Q^{2} \gtrsim 0.2 \mathrm{GeV}^{2}$, in which RPA is practically without influence is of great interest. It allows one to single out the need for the $n p-n h$ contribution without any interference from the RPA effects. At low $Q^{2}$ where the bare description without $n p-n h$ is able to reproduce the data the RPA quenching is then needed to compensate the enhancement from the $n p-n h$ contribution. We remind that in the absence of quantitative evidence in the electron scattering data for the collective nature or the Lorentz-Lorenz quenching, the parameters that govern this effect are not in full control. The neutrino experiments will be of great help to narrow their range.

On the experimental side the same differential cross section but for neutral currents was recently published [2]. It is interesting to compare it to our predictions. Here the final lepton, a neutrino, is not observed and the transfer variable $Q^{2}$ is obtained indirectly from the kinetic energy of the ejected nucleons. In this case it is not quite clear how the multinucleon component shows up in the experimental data. However, the same problem of the axial mass also seems to emerge from these data $[19,20]$. We have thus confronted our theory with the published $Q^{2}$ distribution. The data are for $\mathrm{CH}_{2}$ instead of pure carbon as in our theory, but the difference between the two cases was shown to be small [19]. The comparison of our evaluation with data is shown in Fig. 11. It turns out that the combination of RPA quenching and the $2 p-2 h$ piece leads to a good agreement with data.

\section{CONCLUSIONS}

In conclusion we have investigated in this work more in detail the neutrino- ${ }^{12} \mathrm{C}$ cross section in connection with MiniBooNE data. The most significant quantity is the double differential cross section, which does not imply any reconstruction of the neutrino energy. To compare our theoretical model to these data we have improved our original description applying relativistic corrections. The agreement of our RPA approach with data is quite good once the $n p$ - $n h$ component is included. It confirms our first suggestion that there is no need for a change in the axial mass once the multinucleon processes are taken into consideration. A good agreement is also found for the (single) differential cross sections integrated over one variable where the relativistic corrections play practically no role. We have also examined the $Q^{2}$ distribution, which establishes the necessity of the multinucleon contribution, independently of the RPA quenching. The same description appears to be efficient for the $Q^{2}$ distribution in the case of neutral currents, although the role of the multinucleon component in these experimental data is not obvious. Understanding in detail the role of nuclear dynamics in neutrino-nucleus interactions is important for the neutrino oscillation programs (see, e.g., Ref. [21]) but beyond the question of the axial mass, which is our main goal, our study also has an interest from a purely hadronic viewpoint. The fact that a signature for the RPA influence in the form of the Lorentz-Lorenz quenching, a long-sought-after effect, seems to emerge from neutrino reactions is an additional and unexpected outcome of our study.
[1] A. A. Aguilar-Arevalo et al. (MiniBooNE Collaboration), Phys. Rev. D 81, 092005 (2010).

[2] A. A. Aguilar-Arevalo et al. (MiniBooNE Collaboration), Phys. Rev. D 82, 092005 (2010).

[3] M. Martini, M. Ericson, G. Chanfray, and J. Marteau, Phys. Rev. C 80, 065501 (2009).

[4] M. Martini, M. Ericson, G. Chanfray, and J. Marteau, Phys. Rev. C 81, 045502 (2010).

[5] O. Benhar, P. Coletti, and D. Meloni, Phys. Rev. Lett. 105, 132301 (2010).

[6] J. E. Amaro, M. B. Barbaro, J. A. Caballero, T. W. Donnelly, and C. F. Williamson, Phys. Lett. B 696, 151 (2011).

[7] J. E. Amaro, M. B. Barbaro, J. A. Caballero, T. W. Donnelly, and J. M. Udias, Phys. Rev. D 84, 033004 (2011).
[8] J. Nieves, I. Ruiz Simo, and M. J. Vicente Vacas, Phys. Rev. C 83, 045501 (2011).

[9] J. Nieves, I. R. Simo, and M. J. V. Vacas, arXiv:1106.5374 [hep-ph].

[10] A. Bodek, H. Budd, and M. E. Christy, Eur. Phys. J. C 71, 1726 (2011).

[11] A. Meucci, M. B. Barbaro, J. A. Caballero, C. Giusti, and J. M. Udias, Phys. Rev. Lett. 107, 172501 (2011).

[12] M. B. Barbaro, A. De Pace, T. W. Donnelly, and A. Molinari, Nucl. Phys. A 596, 553 (1996).

[13] A. De Pace, Nucl. Phys. A 635, 163 (1998).

[14] W. M. Alberico, M. Ericson, and A. Molinari, Annals Phys. 154, 356 (1984).

[15] E. Oset and L. L. Salcedo, Nucl. Phys. A 468, 631 (1987). 
[16] J. Delorme and M. Ericson, Phys. Lett. B 156, 263 (1985).

[17] M. Ericson, A. Figureau, and C. Thevenet, Phys. Lett. B 45, 19 (1973).

[18] W. M. Alberico, M. Ericson, and A. Molinari, Nucl. Phys. A 379, 429 (1982).
[19] O. Benhar and G. Veneziano, Phys. Lett. B 702, 433 (2011).

[20] A. V. Butkevich and D. Perevalov, Phys. Rev. C 84, 015501 (2011).

[21] E. Fernandez-Martinez and D. Meloni, Phys. Lett. B 697, 477 (2011). 